\title{
Tolerance Analysis and Synthesis by Means of Deviation Domains, Axi-Symmetric Cases
}

\author{
M. Giordano, S. Samper, J. P. Petit \\ LMECA (Laboratoire de Mécanique Appliquée) \\ Ecole Supérieure d'Ingénieurs d'Annecy, \\ Université de Savoie, BP 806, 74016 Annecy cedex 16, France \\ Max.Giordano@esia.univ-savoie.fr
}

\begin{abstract}
The small displacement torsors are generally used for the representation of the geometrical deviations. The standardised tolerances can then be translated by a set of inequalities between the components of a deviation torsor. In the case of cylindrical tolerance zones, and surfaces of revolution, the inequalities are quadratic and the stack up tolerances are more difficult to calculate. But the axi-symmetric case makes it possible to reduce the space to three dimensions at the maximum instead of six in the general case. Topological operations like the Minkowski sum to carry out the domains for stack up analyse of tolerances are then reduced to operations on polyhedrons. Moreover, we propose a way of taking into account the size tolerances. The first presented application relates to metro-logic inspection for a specification with maximum material condition on both the toleranced surface and the datum. The second example makes it possible to determine the deviation between two surfaces belonging to two different parts after mating them by two contact features.
\end{abstract}

Keywords: tolerance analysis, tolerance synthesis, axi-symmetry, domains.

\section{INTRODUCTION}

The small displacement torsor is used for the modelisation of the geometrical deviation of mechanical parts [Bourdet et al., 1996], [Desrochers et al., 2003].

The components of a small displacement torsor can also be seen as differential parameters for orientation and location. First, it is necessary to precise what is the deviation torsor.

\subsection{Deviation torsor}

A position or orientation tolerance can be translated by the limitations on the components of the deviation torsor. The precise definition of the deviation torsor can be achieved on the following form: a theoretical element is associated to the real toleranced feature (surface or axis). A frame is attached to this theoretical feature. We call it

85

J.K. Davidson (ed.), Models for Computer Aided Tolerancing in Design and Manufacturing, 85-94.

() 2007 Springer. 
toleranced frame. A datum frame is built from one or more other features. The displacement from the datum frame to the toleranced frame can be seen as the sum of a displacement that define the position and orientation of the toleranced feature compared to the datum frame, and an other displacement that characterise the deviation. The deviation torsor is the first order approximation of this deviation displacement.

The datum frame can be built from only one elementary feature like a plane or the axis of a cylinder, but it is also possible to build a datum frame from different features: common datum or ordered datum system. More generally, the different possibilities allowed by the standard can be modelised by the deviation torsor model.

The datum frame and the frame attached to the toleranced feature can have some degrees of freedom. The components of the deviation torsor corresponding to these degrees of freedom cannot be measured and are indefinite values. They can also be considered as zero because there is no deviation for this degrees of freedom. In the same way a size tolerance between two parallel planes in the nominal configuration can be modelised thank to a deviation torsor.

The deviation torsor can be expressed by two vectors for a given point $\mathrm{O}$. The vectorial expression of the torsor is noted $\mathbf{E}=(\boldsymbol{\delta} \boldsymbol{\theta}, \boldsymbol{\delta} \mathbf{O})$ where $\delta \boldsymbol{\theta}$ is the vector for the infinitesimal rotation and $\boldsymbol{\delta} \mathbf{O}$ for the linear displacement of the point $\mathrm{O}$.

For a given frame (i) constituted by a point $\mathrm{O}_{\mathrm{i}}$ and a vectorial base $\mathbf{x}_{\mathbf{i}}, \mathbf{y}_{\mathrm{i}}, \mathbf{z}_{\mathbf{i}}$, the torsor can be written under a scalar form $\mathrm{E}_{\mathrm{i}}=\left(\mathrm{rx}_{\mathrm{i}}, \mathrm{ry}_{\mathrm{i}}, \mathrm{rz}_{\mathrm{i}}, \mathrm{tx}_{\mathrm{i}}, \mathrm{ty} \mathrm{y}_{\mathrm{i}}, \mathrm{z}_{\mathrm{i}}\right)$ where the three first terms are the components of the rotation vector and the others are the components of the displacement of the point $\mathrm{O}_{\mathrm{i}}$.

The great advantage of the torsors for the displacement modelisation is due to the fact that the set of torsors is a vectorial space (dimension 6). The composition of small displacements is obtained by a sum of torsors. This operation is commutative and simple to compute, while the composition of two displacements in the general case (great displacements) is a non commutative operation. For the operations of tolerance transfer or stack up tolerances of assembled parts, the deviations have to be composed. The sum of torsors allows to modelise these operations.

\subsection{Deviation and clearance domain}

A dimensional or geometrical tolerance compatible with the ISO or ANSI standard make it possible to limit the deviation torsor. Considering that any point of the theoretical feature must be inside the tolerance zone, we obtain inequalities on the components of the deviation torsor.

In the configuration space of dimension 6 , a point corresponds to a particular value of the torsor. Therefore, the set of the deviation torsor components according to the tolerance is a domain in this configuration space. The boundary of this domain is a hyper-surface. The equation of these hyper-surfaces on an implicit form are obtained by replacing the inequalities by equalities.

The use of the configuration space to modelise the tolerances has been used by different authors although they are often introduced with different forms. 
Desrochers use the screw parameters or torsor components of small displacements. The position and orientation of a feature is describe by these parameters [Desrochers et al., 2003].

Sacks and Jokowicz, [Sacks and Joskowicz, 1998] describe a tolerance analysis method that computes tolerance zones of parts in configuration space. But the method is limited to planar kinematical systems.

Mujezinovic and Davidson use Tolerance maps, a convex volume of points corresponding to different possible positions of toleranced feature. The study is limited to plane faces [Mujezinovic et al., 2004] and to line or axis [Davidson and Shah, 2002]. The areal coordinate are introduce to describe any configuration of the feature in its tolerance zone as a linear combination of particular configurations. But in fact areal coordinate for position and orientation are the components of small displacement torsor.

The proposed model allows to translate the tolerance zone into a domain in the configuration space those mathematic representation is a set of inequalities on differential parameters. This model enables stack up analysis in an assembly in the worst case. It is the only model in my knowledge that permits to do this on a systematic method even if the computation are complex in the general case [Petit, 2004].

The clearance in a joint between two parts, can also be modelised by a small displacement torsor called clearance torsor. A frame is attached to each part of the joint so that the frames coincide for a particular configuration. For example for a cylindrical joint the particular configuration is obtained when the two axes are in coincidence. The clearance torsor represent the relative displacement of the frames permitted by the clearance in the joint. The clearance domain is also defined in the configuration space. The contact conditions give the inequalities associated to this domain.

The torsor components depends on the frame chosen to express the torsor on a scalar form. We call it the projection frame. It must not be confused with the datum frame used to define the torsor itself. Therefore, the domain depends also of the projection frame. In particular cases, the domain has some characteristics of symmetry, so that the domain is invariant for particular changes of the projection frame. The case of a symmetry of revolution for the geometry and the tolerances is investigated in detail in this paper. But previously, the general case of change the projection frame is considered.

\subsection{Change the projection frame for a torsor}

The frame (0) is constituted by the point $\mathrm{O}_{0}$ and a vectorial base $\mathbf{x}_{0}, \mathbf{y}_{0}, \mathbf{z}_{0}$. The scalar representation of a torsor $E$ in this frame is noted $E_{0}=\left(R_{0}, T_{0}\right)$, where $R_{0}=\left(r x_{0}, r y_{0}, r z_{0}\right)^{T}$ is the colon matrix of the co-ordinate of the small rotation vector in the vectorial base $(0)$.

The frame (1) is constituted by the point $\mathrm{O}_{1}$ and a vectorial base $\mathbf{x}_{1}, \mathbf{y}_{1}, \mathbf{z}_{1}$. The transfer matrix from the base (0) to (1) is noted $\mathrm{P}_{01}$, and $\mathrm{U}_{01}$ is the colon matrix of the co-ordinates of the point $\mathrm{O}_{1}$ in the frame $(0)$.

We note $E_{1}=\left(R_{1}, T_{1}\right)$ the components of the same torsor $E$ but defined in the projection base (1). Then the following matrix expressions give the new components of the torsor: 
$\mathrm{R}_{1}=\mathrm{P}_{10} \mathrm{R}_{0} \quad$ and $\quad \mathrm{T}_{1}=\mathrm{P}_{10} \mathrm{~T}_{0}+\hat{\mathrm{U}}_{10} \mathrm{R}_{0}$

With $\mathrm{P}_{10}=\mathrm{P}_{01}{ }^{-1}=\mathrm{P}_{01}{ }^{\mathrm{T}}$ and $\hat{\mathrm{U}}_{10}$ is the anti-symmetric matrices of the cross-product, associated to the colon matrix: $\mathrm{U}_{10}=-\mathrm{P}_{10} \mathrm{U}_{01}$.

If $\mathrm{U}_{01}=(\mathrm{x}, \mathrm{y}, \mathrm{z}) \quad$ then $\quad \hat{U}_{10}=\left[\begin{array}{llr}0 & -\mathrm{z} & \mathrm{y} \\ \mathrm{z} & 0 & -\mathrm{x} \\ -\mathrm{y} & \mathrm{x} & 0\end{array}\right]$

\section{AXI-SYMMETRIC CASES}

A toleranced system is considered as axi-symmetric when:

- all the functional surfaces taken into account in the tolerances are surfaces of revolution around the same axis $\mathbf{z}$,

- the tolerance zones corresponding to the given tolerances are volumes of revolution around the same axis $\mathbf{z}$.

In consequence, the datum frames are centred on the axis of revolution, and the components of the deviation torsors for the rotation around the axis are always zero. Figure (1a) shows some examples of axi-symmetric geometric tolerances.

The deviation torsors have the following form: $E=(r x, r y, 0, t x, t y, t z)$. Different parts can be assembled. The contact surfaces are surfaces of revolution. The clearance torsor is in the form : $\mathrm{J}=(\mathrm{rx}, \mathrm{ry}, \mathrm{wz}, \mathrm{tx}, \mathrm{ty}, \mathrm{tz})$. The component $\mathrm{wz}$ is the rotation around the axis and is not limited because it is a degree of freedom of the mechanism. The other components are limited by the contact conditions.

When the projection frame is changed, by a rotation of an angle $\theta$, around the $z$ axis, and a translation $\mathrm{z}$ along the same axis, the matrices that define the change of frame are:

$$
\mathrm{U}_{01}=(0,0, \mathrm{z}) \quad \text { et } \quad \mathrm{P}_{01}=\left[\begin{array}{ccc}
\operatorname{Cos} \theta & -\operatorname{Sin} \theta & 0 \\
\operatorname{Sin} \theta & \operatorname{Cos} \theta & 0 \\
0 & 0 & 1
\end{array}\right]
$$

Then the components of a torsor in the frame (1) can be expressed from the components in the frame $(0)$.

$\mathrm{rx}_{1}=\operatorname{Cos} \theta \mathrm{rx}_{0}+\operatorname{Sin} \theta \mathrm{ry}_{0} \quad \mathrm{tx}_{1}=\operatorname{Cos} \theta \mathrm{tx} \mathrm{x}_{0}+\operatorname{Sin} \theta \mathrm{ty}_{0}+\mathrm{zry} \mathrm{ry}_{1}$
$\mathrm{ry}_{1}=-\operatorname{Sin} \theta \mathrm{rx}_{0}+\operatorname{Cos} \theta \mathrm{ry}_{0} \mathrm{ty}_{1}=-\operatorname{Sin} \theta \mathrm{tx} \mathrm{x}_{0}+\operatorname{Cos} \theta \mathrm{ty}_{0}-\mathrm{zrx}_{1}$
$\mathrm{tz}_{1}=\mathrm{tz}_{0}$

\subsection{Case of a cylindrical tolerance zone around the axis of revolution $\mathrm{Oz}$}

We find this case for example for a coaxiality of a cylinder or of any surface of revolution when the datum is an axis built from one or more surfaces of revolution around the same axis.

The deviation torsor is in the form $E=(r x, r y, 0, t x, t y, 0)$ in a frame $\mathrm{O}, \mathbf{x}, \mathbf{y}, \mathbf{z}$. The displacement vector of the points $\mathrm{P}_{1}$ et $\mathrm{P}_{2}$ at the extremity of the axis:

$\delta \mathbf{P}_{1}=(\mathrm{tx}+\mathrm{ry} \mathrm{h} / 2) \mathbf{x}+(\mathrm{ty}-\mathrm{rx} \mathrm{h} / 2) \mathbf{y}$ 
$\boldsymbol{\delta} \mathbf{P}_{2}=($ tx $-\mathrm{ry} \mathrm{h} / 2) \mathbf{x}+($ ty $+\mathrm{rx} \mathrm{h} / 2) \mathbf{y}$

For the model, the interpretation of the coaxiality tolerance leads these two points to be inside the circles $C_{1}$ and $C_{2}$ respectively, at the extremity of the cylindrical tolerance zone. The diameter of this cylinder is $t$ and is length is h. (Fig.1b) So the tolerance is translated by the inequalities:

$$
\begin{aligned}
& (2 \mathrm{tx} / \mathrm{h}+\mathrm{ry})^{2}+(2 \text { ty } / \mathrm{h}-\mathrm{rx})^{2}<(\mathrm{t} / \mathrm{h})^{2} \\
\text { and } \quad & (2 \mathrm{tx} / \mathrm{h}-\mathrm{ry})^{2}+(2 \mathrm{ty} / \mathrm{h}+\mathrm{rx})^{2}<(\mathrm{t} / \mathrm{h})^{2}
\end{aligned}
$$

\subsection{Case of toleranced plane perpendicular to the axis in the nominal configuration}

The tolerance zone is also a cylinder but the value $t$ of the tolerance is the thickness of the cylinder while the diameter $\mathrm{D}$ is the one of the circle that delimits the face. It can be an orientation tolerance, the datum is then an axis, or a position tolerance. In that case the datum defines an axis and a point on this axis. Let $\mathrm{P}$ be a point on the plane face, belonging to the circle (fig. 1c). The polar co-ordinates of this point are $(\mathrm{D} / 2, \theta)$. The projection frame is centred on the $\mathbf{z}$ axis. The co-ordinates of the deviation torsor in this frame is (rx, ry, $0, \mathrm{tx}, \mathrm{ty}, \mathrm{tz})$.

The displacement of the point $\mathbf{P}$ is $\delta \mathbf{P}=(0,0, \mathrm{tz}+\mathrm{rx} \operatorname{Sin} \theta-\mathrm{ry} \operatorname{Cos} \theta)$. This point must be inside the tolerance zone so that :

$(r x \operatorname{Sin} \theta-r y \operatorname{Cos} \theta)<\mathrm{t} / \mathrm{D}-2 \mathrm{tz} / \mathrm{D} \quad$ and $\quad(-\mathrm{rx} \operatorname{Sin} \theta+\mathrm{ry} \operatorname{Cos} \theta)<\mathrm{t} / \mathrm{D}+2 \mathrm{tz} / \mathrm{D}$

These inequalities must be satisfied for all the possible values of $\theta$.

For any value of $t z$, each inequality is satisfied for all the points of a half space limited by a straight line at the constant distance from the origin. The intersection of all these half spaces is limited by the envelope of all the straight lines. Therefore, it is a circle. Its diameter is equal to $(t / D-2 t z / D)$ if $t z$ is positive and $(t / D+2 t z / D)$ if $t z$ is negative. In all the cases, $t z$ is in the interval $[-t / 2, t / 2]$.

The deviation domain is then defined by the following quadratic inequalities:

$(\mathrm{ry})^{2}+(\mathrm{rx})^{2}<(\mathrm{t} / \mathrm{D}-2 \mathrm{tz} / \mathrm{D})^{2}$ and $(\mathrm{ry})^{2}+(\mathrm{rx})^{2}<(\mathrm{t} / \mathrm{D}+2 \mathrm{tz} / \mathrm{D})^{2}$

For an orientation tolerance, only the angular components are limited:

$(\mathrm{ry})^{2}+(\mathrm{rx})^{2}<(\mathrm{t} / \mathrm{D})^{2}$

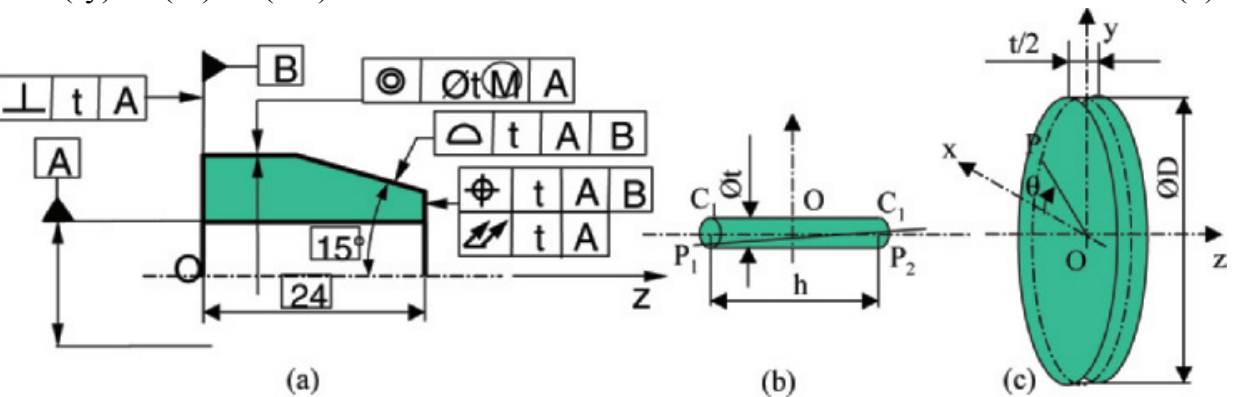

Figure 1; Axi-symmetric cases and two examples of tolerance zones

The general case of the different inequalities can be written on the form: 
$(\mathrm{a} \text { tx }+\mathrm{ry})^{2}+(\mathrm{a} \text { ty }-\mathrm{rx})^{2}<(\mathrm{t} / \mathrm{b}-\mathrm{ctz})^{2}$ and D:

The three previous cases correspond to the following values of the coefficient $a, b$

$$
\begin{aligned}
& \text { 1.) } \mathrm{a}=2 / \mathrm{h} ; \mathrm{b}=\mathrm{h} ; \mathrm{c}=0 \quad \text { and } \mathrm{a}=-2 / \mathrm{h} ; \mathrm{b}=\mathrm{h} ; \mathrm{c}=0 \\
& \text { 2.) } \mathrm{a}=0 ; \quad \mathrm{b}=\mathrm{D} ; \mathrm{c}=2 / \mathrm{D} \quad \text { and } \mathrm{a}=0 ; \quad \mathrm{b}=\mathrm{D} ; \mathrm{c}=-2 / \mathrm{D} \\
& \text { 3.) } \mathrm{a}=0 ; \quad \mathrm{b}=\mathrm{D} ; \mathrm{c}=0
\end{aligned}
$$

\subsection{Axi-symetric domain properties}

The domain remains unchanged if the projection frame rotates around the $\mathrm{z}$ axis and translate on the $\mathrm{z}$ axis.

Let us consider the general inequality:

$\left(\mathrm{a} \mathrm{tx} \mathrm{x}_{1}+\mathrm{ry}_{1}\right)^{2}+\left(\mathrm{a} \mathrm{ty} \mathrm{y}_{1}-\mathrm{rx}_{1}\right)^{2}<\left(\mathrm{t} / \mathrm{b}-\mathrm{ctz}_{1}\right)^{2}$

Then, using the equations (2) and after development and simplifications, the inequality becomes:

$\left(\mathrm{a} \mathrm{tx} \mathrm{x}_{0}+\mathrm{ry}_{0}\right)^{2}+\left(\mathrm{a} \mathrm{ty}_{0}-\mathrm{rx}_{0}\right)^{2}<\left(\mathrm{t} / \mathrm{b}-\mathrm{ctz}_{0}\right)^{2}$

This property proves that the deviation domain remains without change. The deviation domains have a symmetry of revolution in their own configuration space. But it is not the usual symmetry of revolution for figures in the $3 \mathrm{D}$ space. The deviation domains defined by a set of inequalities like in (6) can be represented in a five dimension space because they have only five components or less. The intersection of these domains by an hyper-plane defined by the equations tx $=$ constant, ry=constant and $\mathrm{tz}=$ constant is a domain in the $2 \mathrm{D}$ space. It can be represented in a plane by the domain inside two parallel lines, symmetrical around the origin. The cartesian inequalities for this domain are:

$$
\text { a ty }-\mathrm{rx}<\mathrm{f}(\mathrm{tx}, \mathrm{ry}, \mathrm{tz}) \quad \text { and } \quad \text { a ty }-\mathrm{rx}>-\mathrm{f}(\mathrm{tx}, \mathrm{ry}, \mathrm{tz})
$$

The slope between the "ty" axis and these lines is the coefficient a.

The cross section of an axi-symmetric domain defined by one inequality (6) with an hyper-plane, where tx, ty and tz are constant, is the disk inside the circle of radius $\left(\mathrm{t} / \mathrm{b}-\mathrm{ctz} \mathrm{z}_{0}\right)$. If the domain is defined by several inequalities, the cross section of the domain is the intersection of all the disks built from each inequality. The cross section with an hyper-plane defined by constant rx, ry and $\mathrm{tz}$ is also a disk if "a" is different from zero. These properties characterise the axi-symmetric domains.

When different parts are assembled, the geometrical deviations stack up. The functional requirements concern generally features not belonging to the same part. In the "worst case" approach, the deviation domain that characterise the functional requirement must be inside the Minkowski sum of the different domains of the stack up. See the references [Teissandier et al., 1999], [Giordano et al., 2001], [Petit, 2004] about the applications of Minkowski sum in tolerancing. It can be proved that the Minkowski sum of two axi-symmetric domains is also an axi-symmetric domain. So, it is sufficient to compute the Minkowski sum only in a three dimensional space. Three components are considered: rx, ty, tz or tx, ry, tz. The quadratic inequality (6) is then replaced by the two linear inequalities (7). 


\section{APPLICATION TO THE INSPECTION OF SPECIFICATIONS}

The example concerns a coaxiality tolerance for the axis of a cylinder. The datum is the axis of an other cylinder. The maximal material modifier is used both for the toleranced feature and the datum (fig.2). The proposed method is quite different from those presented in the literature [Davidson and Shah, 2003].
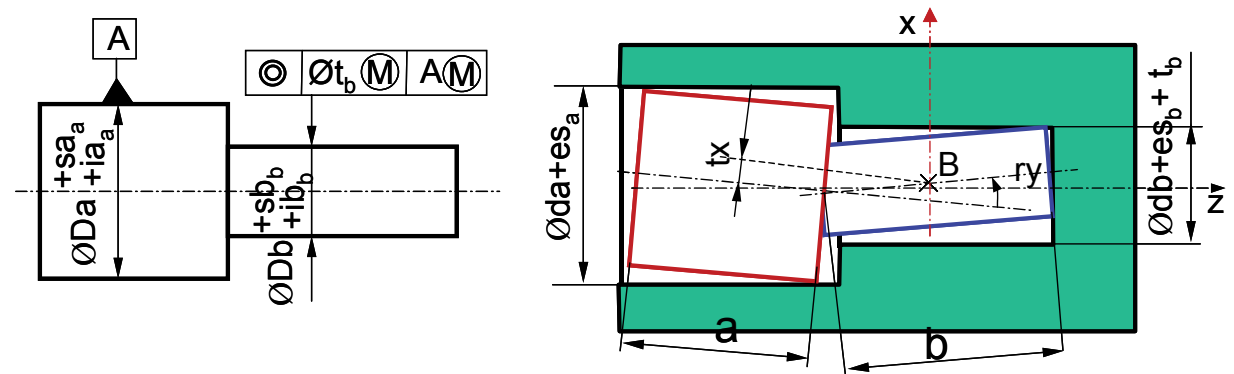

Figure 2; coaxiality tolerance and interpretation

The problem is to determine the relations between the deviation parameters that can be measured (components of the deviation torsors) so that the part is in accordance with the tolerance.

It is assumed that after the measurement, the following parameters are known :

- the diameters of the associated cylinders : $(\mathrm{Da}+\mathrm{ea})$ and $(\mathrm{Db}+\mathrm{eb})$,

- the deviation components for the rotation and the position of the cylinder B with respect to the cylinder A : rx,ry, tx and ty.

We suppose that the dimensional tolerances are defined :

ia $<$ ea $<$ sa, and $\mathrm{ib}<\mathrm{eb}<\mathrm{sb}$,

and the coaxiality tolerance specified value is tb.

The part is in conformity with the specifications if the tolerances of size are checked and if the part can be assembled in its theoretical gauge. For the gauge, the dimension of the diameter of the cylinder $\mathrm{B}$ is the maximum value added to the geometric tolerance $\mathrm{Db}+\mathrm{sb}+\mathrm{tb}$, while the diameter of $\mathrm{A}$ is the maximum value $\mathrm{Da}+$ sa. We consider the mechanism constituted by the part assembled in the gauge. The subscript (0) is for the gauge and (1) for the part.

Since the two cylindric joints A and B and the two parts constitute a single loop mechanism, the clearance and deviation torsors are linked by the relation:

$\mathrm{J}_{0 \mathrm{~A} 1}+\mathrm{E}_{\mathrm{A} 1 \mathrm{~B}}+\mathrm{J}_{1 \mathrm{~B} 0}+\mathrm{E}_{\mathrm{B} 0 \mathrm{~A}}=0$

Where $\mathrm{J}_{0 \mathrm{~A} 1}$ and $\mathrm{J}_{1 \mathrm{~B} 0}$ are the clearance torsors for the two joints and $\mathrm{E}_{\mathrm{A} 1 \mathrm{~B}}$ and $\mathrm{E}_{\mathrm{B} 0 \mathrm{~A}}$ are the deviation torsors of the parts. The geometry of the gauge is perfect, therefore $\mathrm{E}_{\mathrm{B} 0 \mathrm{~A}}=0$.

The equivalence between the geometric tolerance and the assembly is translated into the condition between the domains: $\left[\mathrm{E}_{\mathrm{A} 1 \mathrm{~B}}\right]=\left[\mathrm{J}_{0 \mathrm{~B} 1}\right]+\left[\mathrm{J}_{1 \mathrm{~A} 0}\right]$. The sum of Minkowski of the domain. The domain is built in the plane rx, ty (fig.3). 
The domain $\left[\mathrm{J}_{1 \mathrm{~A} 0}\right]$ is determined at the point $\mathrm{B}$. The parameters that characterize the domain $\left[\mathrm{E}_{\mathrm{A} 1 \mathrm{~B}}\right]$ allows to write the inequalities between the deviation parameters.

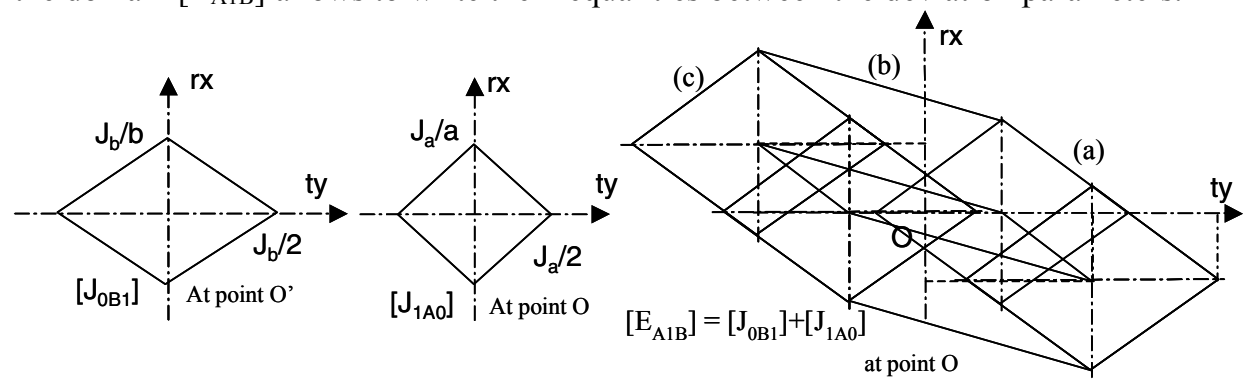

Figure 3 ; Deviation domain and their Minkowski sum

\section{APPLICATION TO TOLERANCE SYNTHESIS}

In the second example the problem is to compare the deviation resulting from the assembly of two parts, with a requirement between two feature belonging to the different parts. The parts are toleranced in a qualitative form but the values of the tolerance zones are unknown. The qualitative tolerances are defined in figure 4. They are justified by technological choices for the joint between the two parts. The head 2 is in contact with the barrel 1, on the plane A maintained by tie rods and is centred in the "short" cylinder C fitted with a clearance in the cylinder B.

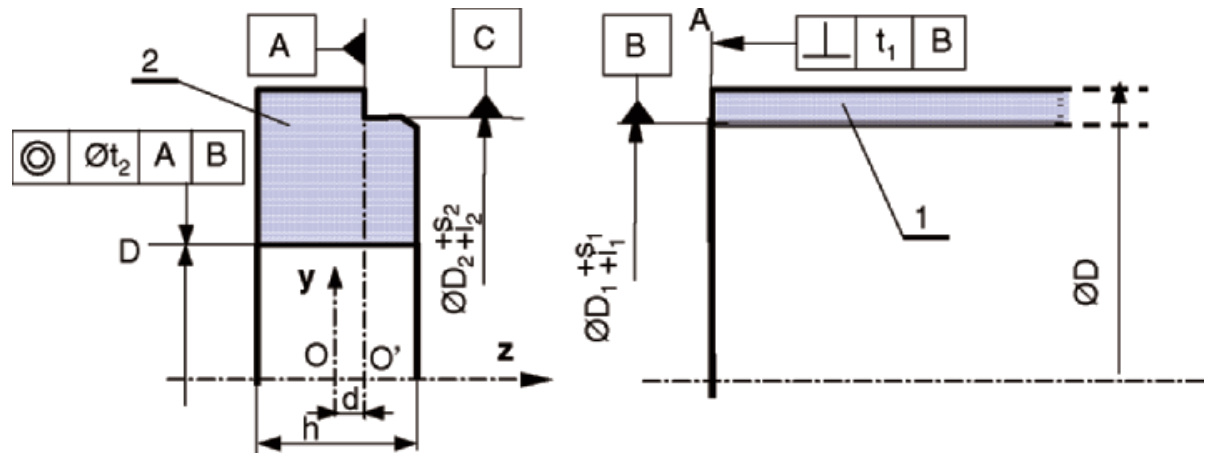

Figure 4; Qualitative tolerances of axi-symmetric parts for tolerance synthesis

We notice :

- B: a frame attached to the cylinder B,

- (1): a frame attached to the plane A but centred on the B axis of the part (1),

- (2): a datum ordered frame attached to the part (2), so that the plane A is the primary datum and cylinder $\mathrm{C}$ is the secondary datum.

- D: the datum attached to the cylinder D. 
The deviation torsors are linked by the relation: $\quad E_{B D}=E_{B 1}+E_{12}+E_{2 D}$

Each torsor represent the small displacements to go from a frame to the other. $\mathrm{E}_{\mathrm{B} 2}$ is the deviation of the plane with regard to the cylinder. The domain $\left[\mathrm{E}_{\mathrm{B} 1}\right]$ is built from the perpendicularity tolerance. It is characterised by the parameter $t_{1}$. The domain is defined at the point $\mathrm{O}^{\prime}$ in the plane ty, rx. If the torsors are expressed at the point $\mathrm{O}$, the domain represented by a segment is changed into an other segment.

$E_{21}$ is the deviation torsor of the frame (2) with regard to the frame (1). This deviation is due to the clearance between the cylinder $\mathrm{C}$ and $\mathrm{B}$. We notice $\mathrm{J}$ the difference between the two diameters of the cylinders.

Since the planes A of the two parts are in coincidence, the relative displacement is a translation in the plane. The domain $\left[\mathrm{E}_{12}\right]$ is independent on the point chosen to define the torsor. $\left[E_{2 D}\right]$ is the deviation domain for the $\mathrm{D}$ toleranced feature. The datum frame is in fact the frame (2). It is defined at the point $\mathrm{O}$. The three domains are represented in the figure 5 .

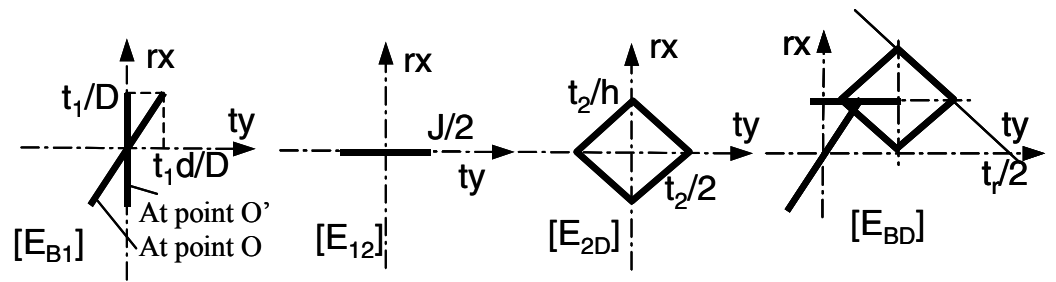

Figure 5; Deviation domains and stack up tolerances

The three deviation torsors have any value in their domain. When the parts are assembled the torsor sum $\mathrm{E}_{\mathrm{BD}}$ will belong to the domain obtained by the Minkowski sum of the three previous domains defined at the same point $\mathrm{O}$.

The resulting domain does not correspond to a tolerance zone. The stack up tolerance of geometric standardised tolerances is not in general case a standardised tolerance. Nevertheless, if the functional requirement between the feature B and D is given using a coaxiality tolerance, it is possible to determine the relation between the value $t_{r}$ of this tolerance and the values of the intermediate tolerances. The relation is easy to determine thanks to the domain operations in the plane rx, ty (fig.5) :

$\mathrm{t}_{\mathrm{r}}=\mathrm{t}_{1}(\mathrm{~h}+2 \mathrm{~d}) / \mathrm{D}+\mathrm{J}+\mathrm{t}_{2}$

Different solutions are possible. Others criteria must be given for the repartition of the three values $J, t_{2}$ and $t_{1}$. Since $J$ depends of the actual size, the worst case is when $J$ is maximum, so the clearance has a unfavourable effect on the requirement. The least material condition can then be used.

\section{CONCLUSION}

The method based on deviation and clearance domain is very efficient for the tolerance analysis. Tolerance synthesis needs to compute Minkowski sum on the domains. In the 
general case the operation is complex but in the case of axi-symmetric systems, the problem can be solved with simple linear operations. From qualitative tolerances, it is possible to determine analytic relations that permits to determine the tolerances that ensure given requirements. This synthesis tolerancing approach can be applied to different practical systems.

\section{REFERENCES}

[Bourdet et al., 1996] Bourdet P., Mathieu L., Lartigue C., Ballu A., «The concept of small displacement torsor in metrology» Advanced mathematical tool in metrology, Advances in mathematics for applied sciences, World Scientific, Vol 40, 1996.

[Desrochers et al., 2003] Desrochers A., Béron V. and Laperrière L., "Revisiting Screw Parameter Formulation for Accurate Modeling of Planar Tolerance Zones », the $8^{\text {th }}$ international CIRP Seminar on Computer Aided Tolerancing, Charlotte, North Carolina, USA, 28-29 April 2003, pp.239-248.

[Sacks and Joskowicz, 1998] L., Sacks E., Joskowicz L., "Parametric kinematic tolerance analysis of general planar systems. Computer-aided design 1998, vol 30(9), pp. 707-714.

[Mujezinovic et al., 2004] Mujezinovic A., Davidson J.K., Shah J.J., "A New Mathematical Model for Geometric Tolerances as Applied to Polygonal Faces ", Transaction of the ASME, J. of Mech. Design, Vol. 126, May 2004, pp. 504-518.

[Davidson and Shah, 2002] Davidson J.K, and Shah J.J, «Geometric tolerances : a new application for line geometry and screws » ImechE Journal of Mechanical Engineering Sciences, Vol.216, Part C, 2002, pp.95-104.

[Petit, 2004] Petit J.P., « Spécification géométrique des produits : méthode d'analyse de tolérances. Application en conception assistée par ordinateur. » P.h.d. theses, Université de Savoie, France, 17 déc. 2004.

[Teissandier et al., 1999] Teissandier D., Delos V., Couetard Y., "Operations on polytopes: application to tolerance analysis", 6th CIRP inter. Seminar on computeraided tolerancing, Univ. of Twente, Enschede, The Netherlands, 22-24 march 1999.

[Giordano et al., 2001] Giordano M., Kataya B., Pairel E., «Tolerance analysis and synthesis by means of clearance and deviation spaces », Geometric product Specifcation and verification, selected conf. papers of the $7^{\text {th }}$ CIRP seminar on Computer Aided Tolerancing, April 2001, Kluwer Academic Pub., pp.145-154.

[Davidson and Shah, 2003] Davidson J.K. and Shah J.J., «Using Tolerance-Maps to represent material condition on both a feature and a datum $»$, the $8^{\text {th }}$ international CIRP Seminar on Computer Aided Tolerancing, Charlotte, North Carolina, USA, 28-29 April 2003, pp.92-101. 